\title{
A SPECIAL CASE OF THE NORMAL DERIVATIVE PROBLEM FOR A THIRD ORDER COMPOSITE PARTIAL DIFFERENTIAL EQUATION ${ }^{1}$
}

\author{
ROBERT B. DAVIS
}

Composite equations have been discussed in $[2 ; 4 ; 7 ; 8]^{2}$ and in [3, pp. 31-32]. The appearance of third order composite equations in hydrodynamics is mentioned briefly in [2]. More important, in [6, pp. 122-123] it is desired to study solutions of a fourth order equation of hydrodynamics [6, equation (3.5)] in the limiting case when the coefficients of the fourth order terms become zero. One is thus led to inquire into the relationship between solutions of this fourth order equation, and those of the resulting equation of the third order obtained by deleting the fourth order terms. This, in turn, suggests the investigation of problems such as that of the present paper. (Discussion of problems of the "reduction of order" type, using separation of variables and ordinary differential equations, is given in [6] and in the works of Wasow [for example, 10]. More recent work along these lines is found in [9] and in some unpublished results of R. C. DiPrima and C. C. Lin.)

In the present paper, we prove an existence theorem for a certain boundary value problem, for the differential equation

$$
\frac{\partial}{\partial x}(\Delta u)=\Delta\left(\frac{\partial u}{\partial x}\right) \equiv \frac{\partial^{3} u}{\partial x^{3}}+\frac{\partial^{3} u}{\partial y^{2} \partial x}=0 .
$$

Note that equation (1) is composite, with the lines $y=$ constant for characteristics.

THEOREM. Let $y_{2} \geqq y_{1} \geqq 0$, and let $S_{1}$ denote the segment of the $y$-axis $-y_{1} \leqq y \leqq y_{1}, S_{2}$ denoting the longer segment $-y_{2} \leqq y \leqq y_{2}$. Let $A$ be a curve lying in the half-plane $0 \leqq x$, extending from $P_{1}\left(0,-y_{1}\right)$ to $P_{2}\left(0, y_{1}\right)$, and having continuous curvature. We suppose that at $P_{1}$ and $P_{2}$ the first and second derivatives

$$
d y / d x, d^{2} y / d x^{2},
$$

for the curve $A$, are zero. However, we suppose that $A$ intersects the lines

Presented to the Society, October 24, 1953; received by the editors October 9 , 1953 and, in revised form, February 23, 1954.

1 The preparation of this paper has been supported, in part, by the Office of Ordnance Research, U. S. Army, under Contract DA-19-020-ORD-2245.

2 Numbers in brackets refer to the bibliography at the end of the paper. 
$y= \pm y_{1}$ only in the two points $P_{i}, i=1,2$. Let $R$ denote the finite region bounded by $S_{1}$ and $A$.

We suppose that $A$ is such that the region $R$ is convex, with every characteristic of equation (1) in $R$ intersecting $A$ in exactly one point.

Let

$$
g_{1}(x, y)=\sum_{n=1}^{\infty} a_{n}^{1}(x) y^{n} \quad \text { and } \quad g_{2}(y)=\sum_{n=1}^{\infty} a_{n}^{2} y^{n}
$$

where we assume that, for $|y| \leqq y_{2},|x| \leqq y_{2}$, we have $g_{1} \in C^{2}$ in $x$, with $g_{1}, g_{2}$, and all derivatives of $g_{1}$ up to the second order being analytic functions of $y$. Let $M$ be a constant such that

$$
\left|a_{n}^{i}\right| \leqq \frac{M}{y_{2}^{n}}, \quad i=1,2,
$$

for $|x| \leqq y_{2}$. Making $M$ large enough, also, to dominate the coefficients in the series expansions of the other initial values given below in equation (7) (that is, for the functions $y, \partial g_{1} / \partial y, h(y)-\partial^{2} g_{1} / \partial y^{2}(0, y)$, etc.), in this same way, we require that the curve $A$ be such that, for all $(x, y) \in R \cup A$,

$$
|y+11 M x|<y_{2} \text {. }
$$

We assume, finally, that the values prescribed for $u_{x}$ at $P_{1}$ and $P_{2}$ are consistent, that is,

$$
\frac{\partial g_{1}}{\partial x}=g_{2} \quad \text { at } P_{1} \text { and also at } P_{2}
$$

Conclusion: Then there exists a unique function of $u$ such that:

(i) $u \in C^{3}$ in $R$.

(ii) $u \in C$ on $R \cup S_{1} \cup A: u_{x} \in C$ on $R \cup S_{1}$.

(iii) $u$ satisfies equation (1) in $R$.

(iv) $u=g_{1}(x, y)$ on $A \cup S_{1}$.

(v) $\frac{\partial u}{\partial x}=\frac{\partial u}{\partial n}=g_{2}(y)$ on $S_{1}$.

REMARK. It is actually sufficient to hypothesize analytic boundary values on $S_{2}$ only, with twice-differentiable boundary values on $A$. To do so, however, considerably complicates the notation and details of proof.

Proof of THEOREM. We begin by proving that the solvability of 
the boundary value problem described by equations (3), which we refer to as Problem I, is equivalent to the solvability of the following boundary value problem:

PRoblem II.

(i) $u^{*} \in C^{3}$ in $R$.

(ii) $u^{*} \in C$ on $R \cup S_{1} \cup A ; u_{x}^{*} \in C$ on $R \cup S_{1}$.

(iii) $\frac{\partial}{\partial x}\left(\Delta u^{*}\right)=0$.

(iv) $u^{*}=g^{*}$ on $A$ ( $g^{*}$ is defined below, in equation (8)).

(v) $u^{*}=0$ on $S_{1}$.

(vi) $u_{n}^{*}=0$ on $S_{1}$.

(The more specialized boundary values of Problem II are essential to our subsequent use of symmetry.)

Equivalence of Problems I and II: We define $h(y)$ as the linear function of $y$ which takes, at $P_{1}$ and at $P_{2}$, the values

$$
h\left( \pm y_{1}\right)=\Delta g_{1}\left(0, \pm y_{1}\right) .
$$

We now solve an initial value problem, for the unknown function $v(x, y)$, prescribed by equations (5):

Problem III.

$$
\Delta v_{x}=0,
$$

with the initial values, given on $S_{2}$ :

$$
\begin{aligned}
v(0, y) & =g_{1}(0, y), \\
v_{x}(0, y) & =g_{2}(y), \\
v_{x x}(0, y) & =h(y)-\frac{\partial^{2} g_{1}}{\partial y^{2}}(0, y) .
\end{aligned}
$$

The region $R^{*}$ within which $v(x, y)$ satisfies equation (5-1) will be described presently.

From $[1$, p. $35 \mathrm{ff}$.$] , we know that Problem III is equivalent to the$ following partial differential equation system, together with the initial values $(7)$ :

$$
\begin{gathered}
\eta_{x}=0, \quad v_{x}=p \eta_{y}, \quad q_{x}=p_{y}, \quad p_{x}=r \eta_{y} \\
s_{x}=r_{y}, \quad t_{x}=s_{y}, \\
r_{x}=l \eta_{y}, \quad j_{x}=l_{y}, \quad k_{x}=j_{y}, \quad m_{x}=k_{y}, \quad l_{x}=-j_{y} .
\end{gathered}
$$


The following initial values are prescribed on $S_{2}$ :

$$
\begin{gathered}
\eta(0, y)=y, v(0, y)=g_{1}(0, y), q(0, y)=\frac{\partial g_{1}}{\partial y}(0, y), p(0, y)=g_{2}(y), \\
r(0, y)=h(y)-\frac{\partial^{2} g_{1}}{\partial y^{2}}(0, y), s(0, y)=g_{2}^{\prime}(y), t(0, y)=\frac{\partial^{2} g_{1}}{\partial y^{2}}(0, y), \\
j(0, y)=h^{\prime}(y)-\frac{\partial^{3} g_{1}}{\partial y^{3}}(0, y), \quad k(0, y)=g_{2}^{\prime \prime}(y), \\
m(0, y)=\frac{\partial^{3} g_{1}}{\partial y^{3}}(0, y), \quad l(0, y)=-g_{2}^{\prime \prime}(y) .
\end{gathered}
$$

In this new formulation, Problem III becomes a Cauchy-Kowalewski problem [1, p. $39 \mathrm{ff}$.$] .$

As a consequence of our hypotheses, Problem III is solvable for a region $R^{*}$ which contains $R \cup A$, since the dominating functions, $w$, of the Cauchy-Kowalewski method are:

$$
w(x, y)=\frac{y_{2} M}{y_{2}-y-11 M x}
$$

[cf. 1, p. 43], and the $w$ therefore provide suitable domination over the larger region $R^{*}$.

Having thus determined $v(x, y)$ as a solution of Problem III, we see that $u$ and $u^{*}$ are related by:

$$
u^{*}=u-v .
$$

The definition of $g^{*}$ (cf. equations (4)) is given as:

$$
g^{*}(x, y)=g_{1}(x, y)-v(x, y) \text { on } A ;
$$

if we regard $g^{*}(s) \equiv g^{*}(x, y)$ as a function of arc-length along $A$, we find that

$$
g^{*}(s) \in C^{2},
$$

and also, at $P_{1}$ and $P_{2}$,

$$
\begin{aligned}
\frac{d g^{*}(s)}{d s} & =\frac{\partial g^{*}(x, y)}{\partial x} \frac{d x}{d s}+\frac{\partial g^{*}(x, y)}{\partial y} \frac{d y}{d x} \\
& =0 \cdot \frac{d x}{d s}+\frac{\partial g^{*}(x, y)}{\partial y} \cdot 0 \\
& =0
\end{aligned}
$$


and

$$
\begin{aligned}
\frac{d^{2} g^{*}(s)}{d s^{2}}= & g_{x x}^{*}\left(\frac{d x}{d s}\right)^{2}+2 g_{x y}^{*} \frac{d x}{d s} \frac{d y}{d s} \\
& +g_{x}^{*} \frac{d^{2} x}{d s^{2}}+g_{y y}^{*}\left(\frac{d y}{d s}\right)^{2} \\
& +g_{y}^{*} \frac{d^{2} y}{d s^{2}} \\
= & 0 .
\end{aligned}
$$

These properties of $g^{*}(s)$ are also essential to our subsequent use of symmetry.

Reduction to Sjöstrand's problem. We now reduce Problem II to a problem of the type considered by Sjöstrand $[7 ; 8]$. To do this we construct a symmetric region $\widetilde{R}$, as follows. Let $\widetilde{R}$ consist of the region $R$, plus its mirror image in the $y$-axis, plus a certain segment of the $y$-axis; precisely, $\widetilde{R}$ shall consist of all $(x, y)$ such that either $(x, y)$ $\in R$, or else $(-x, y) \in R$, plus the segment of the $y$-axis: $-y_{1}<y<y_{1}$. We extend all functions by the symmetry rule that, for $x<0, f(x, y)$ $\equiv f(-x, y)$; these extended functions are denoted by a tilde, the same notational device being used also for arcs, regions, etc. Thus, $\tilde{g}^{*}(s)$ is the extension to $\tilde{A}$ of the function $g^{*}(s)$ defined on $A$.

Notice that the boundary values for $\tilde{u}^{*}$ are now prescribed around the entire boundary $\widetilde{A}$ of the simply-connected region $\widetilde{R}$, while values for $\tilde{u}^{*}$ and $\partial \tilde{u}^{*} / \partial x$ are prescribed along a curve lying within $\tilde{R}$ (namely, the segment of the $y$-axis contained in $\widetilde{R}$ ). We relax, temporarily, the prescription of $\partial \tilde{u}^{*} / \partial x$ along $S_{1}$. The remaining boundary values, together with the equation

$$
\Delta\left(\tilde{u}_{x}^{*}\right)=0,
$$

constitute precisely the form of boundary value problem considered by Sjöstrand. Moreover, Sjöstrand's hypothesis is satisfied, since $\widetilde{A}$ has continuous curvature and $\tilde{g}^{*}(s) \in C^{2}$ on $\tilde{A}$, including at the points $P_{\boldsymbol{i}}$.

We know, consequently, that $\tilde{u}^{*}$ exists and is unique. From symmetry, moreover, one shows easily that $\tilde{u}^{*}$ satisfies also the requirement that

$$
\partial \tilde{u}^{*} / \partial x=0
$$

on $S_{1}$.

The existence and uniqueness of $u$ follows from that of $\tilde{u}^{*}$. This, then, completes the proof of the theorem. 


\section{BIBLIOGRAPHY}

1. R. Courant and D. Hilbert, Methoden der mathematischen Physik, vol. 2, Berlin, 1937.

2. R. B. Davis, $A$ boundary value problem for third-order linear partial differential equations of composite type, Proc. Amer. Math. Soc. vol. 3 (1952) pp. 751-756.

3. J. Hadamard, Equations aux dérivées partielles. Les conditions définies en générale. Le cas hyperbolique, L'Enseignement Mathématique vol. 35 (1936) pp. 5-42.

4. - Propriêtés d'une équation linéaire aux dérivées partielles du quatrième ordre, Tôhoku Math. J. vol. 37 (1933).

5. N. Levinson, The first boundary value problem for $\epsilon \Delta u+A(x, y) u_{x}+B(x, y) u_{y}$ $+C(x, y) u=D(x, y)$ for small $\epsilon$, Ann. of Math. vol. 51 (1950) pp. 428-445.

6. C. C. Lin, On the stability of two-dimensional parallel flows. Part I. General theory, Quarterly of Applied Mathematics vol. 3 (1945) pp. 117-142.

7. O. Sjöstrand, Sur une équation aux dérivées partielles du type composite, Arkiv för Matematik, Astronomi och Fysik vol. 25 A, No. 21, pp. 1-11.

8. - Sur une équation aux dérivées partielles du type composite. Note 2, Arkiv för Matematik, Astronomi och Fysik vol. 26 A, No. 1 (1939) pp. 1-10.

9. W. Wasow, Asymptotic solution of the differential equation of hydrodynamic stability in a domain containing a transition point, Ann. of Math. vol. 75 (1953) pp. 222-252.

10. - The complex asymptotic theory of a fourth order differential equation of hydrodynamics, Ann. of Math. vol. 49 (1948) pp. 852-871.

University OF NeW Hampshire 